University of Wollongong

Research Online

Faculty of Engineering and Information

Faculty of Engineering and Information

Sciences - Papers: Part A

Sciences

2013

\title{
Modeling and experimental investigation of rotational resistance of a spiral- type robotic capsule inside a real intestine
}

Hao Zhou

University of Wollongong, hz467@uowmail.edu.au

Gursel Alici

University of Wollongong, gursel@uow.edu.au

Trung Duc Than

University of Wollongong, dtt581@uowmail.edu.au

Weihua Li

University of Wollongong, weihuali@uow.edu.au

Follow this and additional works at: https://ro.uow.edu.au/eispapers

Part of the Engineering Commons, and the Science and Technology Studies Commons

Research Online is the open access institutional repository for the University of Wollongong. For further information contact the UOW Library: research-pubs@uow.edu.au 


\title{
Modeling and experimental investigation of rotational resistance of a spiral-type robotic capsule inside a real intestine
}

\begin{abstract}
In this study, the rotational resistance of a spiral-type capsule rotating inside a small intestine is investigated by in vitro experiments and analytical modeling, on which a limited literature is available. The results presented exhibit viscoelastic nature of the intestinal tissue. The significance of various spiral structures and rotating speeds is quantitatively evaluated from the propulsion point of view. Also, an analytical torque model is proposed and subsequently validated. The close match between the experimental results and numerical results from the model shows that the model is reasonably accurate to estimate the rotational resistance torque of the small intestine. Both the experimental and modeling works provide a useful guide to determine the torque required for a spiral-type endoscopic capsule operating in a 'really' small intestine. Therefore, the proposed torque model can be used in the design and optimization of in-body robotic systems, which can remotely be articulated using magnetic actuation.
\end{abstract}

\section{Keywords}

rotational, resistance, spiral, modeling, type, experimental, robotic, capsule, inside, real, intestine, investigation

\section{Disciplines}

Engineering | Science and Technology Studies

\section{Publication Details}

Zhou, H., Alici, G., Than, T. Duc. \& Li, W. (2013). Modeling and experimental investigation of rotational resistance of a spiral-type robotic capsule inside a real intestine. IEEE-ASME Transactions on Mechatronics, 18 (5), 1555-1562. 


\title{
Modeling and Experimental Investigation of Rotational Resistance of a Spiral-Type Robotic Capsule Inside a Real Intestine
}

\author{
Hao Zhou, Gursel Alici, Trung Duc Than, and Weihua Li
}

\begin{abstract}
In this study, the rotational resistance of a spiral-type capsule rotating inside a small intestine is investigated by in vitro experiments and analytical modeling, on which a limited literature is available. The results presented exhibit viscoelastic nature of the intestinal tissue. The significance of various spiral structures and rotating speeds is quantitatively evaluated from the propulsion point of view. Also, an analytical torque model is proposed and subsequently validated. The close match between the experimental results and numerical results from the model shows that the model is reasonably accurate to estimate the rotational resistance torque of the small intestine. Both the experimental and modeling works provide a useful guide to determine the torque required for a spiraltype endoscopic capsule operating in a "really" small intestine. Therefore, the proposed torque model can be used in the design and optimization of in-body robotic systems, which can remotely be articulated using magnetic actuation.
\end{abstract}

Index Terms-.

\section{INTRODUCTION}

$\mathbf{T}$ HE wireless capsule endoscope (WCE) has been universally used as a first-line medical tool to diagnose diseases in the gastrointestinal (GI) tract, especially in the small intestine, where traditional endoscopes are hard to access [1]-[3]. It is believed that more attractive applications of WCE, such as targeted drug delivery and telepresence surgery, will be realized in the near future if a robotic capsule with active locomotion is developed to break its dependence on the natural peristalsis for movement [4].

Significant effort has been dedicated to exploring novel locomotion mechanisms [5]-[8]. Among them, magnetic propulsion is promising since it does not require onboard batteries and the control unit is moved out of the capsule, too. Therefore, much space can be saved, which is a vital advantage for a millisized robotic device or even smaller size. In addition, a limited opera-

Manuscript received January 4, 2012; revised April 16, 2012; accepted July 2, 2012. Recommended by Technical Editor Y. Sun. This work was supported by the Intelligent Nano-Tera Systems Research Laboratory of the University of Wollongong. The work of H. Zhou and T. D. Than was supported by the University of Wollongong Research Council under the Ph.D. scholarships.

The authors are with the School of Mechanical, Materials and Mechatronic Engineering, University of Wollongong, Wollongong, N.S.W., 2522, Australia (e-mail: hz467@uowmail.edu.au; gursel@uow.edu.au; dtt581@uowmail.edu. au; weihuali@uow.edu.au).

Color versions of one or more of the figures in this paper are available online at http://ieeexplore.ieee.org.

Digital Object Identifier 10.1109/TMECH.2012.2208121 tion time will no longer be a problem due to remotely powering of a magnetically propelled robotic device.

Two approaches are generally adopted to implement magnetic propulsion. The first approach [9]-[11] is straightforward, exerting a direct pulling force on an internal magnetic part by producing a magnetic field gradient. However, this pulling force decays rapidly when the gap between the robot and the magnetic source increases. The second approach [12], [13] is based on embedding a magnet with its magnetization lateral to the capsule's axis and then generating a rotating magnetic field so that the capsule can be acted upon by a magnetic torque and hence the capsule spins about its axis. When a spiral structure is mounted on the capsule's surface, the rotation is converted to a translational movement. Compared to the direct-pulling method, the propulsion of a spiral-type robot is advantageous because the maximum torque available to the robot is proportional to the magnetic field intensity, which declines slower than the magnetic field gradient over a long distance. Three-axis Helmholtz coils with three separate sinusoidal current inputs are able to produce a rotating magnetic field with a uniform intensity and an adjustable rotational axis so that the robot is propelled and steered along the curved GI tract [13].

To develop a spiral-type WCE, the geometry of the helical structure must be optimized since it plays a significant role in determining the propulsion efficiency. As a medical microrobot traveling in a deflated, winding, and slippery lumen, the complexity of its working environment makes this optimization problem even more critical. Therefore, the resistant characteristics of the GI tract should be evaluated in order to provide more accurate data with the optimization process.

Recently, some research work has been reported on the biomechanical and tribological properties of the GI tract. Baek et al. measured the frictional resistance of capsules with different shapes and dimensions moving along porcine small intestinal samples and concluded that a smooth cylindrical capsule with a smaller diameter performed better in avoiding translational friction [14]. In their work, they investigated the small intestine's properties further and reported on a viscoelasticity model for the stress relaxation [15] as well as an analytical model [16] to predict the friction from a linear movement of a smooth cylindrical capsule inside the small intestine. Wang and Meng conducted a series of tests with 15 plastic capsules of various diameters and lengths inside the segments of porcine small intestine [17]. The resistant forces from 20 to $100 \mathrm{mN}$ were measured for the capsules which had the diameters in the range of $8-13 \mathrm{~mm}$ and the translational speed of $0.5 \mathrm{~mm} / \mathrm{s}$. Wang and Yan performed 
the tests by pulling a set of specially prepared frictional samples with different surface profiles on planar open intestinal samples [18]. The tests showed that a flat contact surface caused the least resistance while a triangular one led to the most. Terry et al. performed experiments on active forces from the myenteron, biomechanical response, mucus adhesivity, and tribology of the porcine small intestine [19]. By comparing the in vivo and in vitro tribometry tests, it was suggested that the coefficient of friction (COF) might slightly drop as the tissue became dead. Bellini et al. proposed a constitutive model, with parameters identified from planar biaxial test data, to predict biomechanical response of the small bowel under complex loading [20]. All these work has contributed to a better understanding of the mechanical properties of the small intestine for active capsule endoscopes. However, none of them studied the frictional resistance for a spiral-type capsule rotating inside a small intestine, which represents the real operation condition of a robotic capsule. This study aims to close this gap in the literature by establishing a mathematical model to predict the mechanical torque required to rotate the robotic capsule in a viscoelastic environment such as the GI tract.

This paper investigates the rotational resistance of a spiraltype capsule rotating inside the GI tract. From a physiological point of view, this is the resistance which the robotic capsule must overcome in order to start and maintain its rotation. The small intestine of a porcine was employed for the in vitro experiments since its mechanical properties were reported to be quite similar to those of a human being [21]. Four capsules with different spiral structures were tested under various rotating speeds. The rotational resistance was measured and presented in the form of torque. Furthermore, an analytical model was proposed for the prediction of this frictional resistance. Some parameters were identified with one set of experimental results and other sets of experimental results were used to further validate the model. This study provides a useful guide to characterize the required torque for a spiral-type capsule and to undertake the design and optimization of the microrobots' traction topology.

\section{EXPERIMENTS}

\section{A. Experimental Setup}

Dummy Pillcam SB2 capsules (Given Imaging) were adopted as the bases of the microrobots. For each capsule, a segment of wire $(\phi 1 \mathrm{~mm})$ was wounded around the outer surface and acted as the spiral structure. The winding area was within the cylindrical part of the capsule so that every spiral structure had the same dimension $(15 \mathrm{~mm})$ in the longitudinal axis. Four spiral-type capsules of such were fabricated, with the helical angles of $5^{\circ}$ (No. 1 ), $10^{\circ}$ (No. 2), $15^{\circ}$ (No. 3), and $20^{\circ}$ (No. 4), respectively, as shown in Fig. 1. For each of them, only one spiral was attached on the surface.

A steel rod was fixed to one end of the capsule so that the assembly could be connected to a torque sensor, which was able to output real-time measurements to its indicator. Via an RS232 cable and an interface program, the data were consequently sent to a PC for recording. During the tests, the capsule was kept still and the small intestine was spinning instead. The segment was

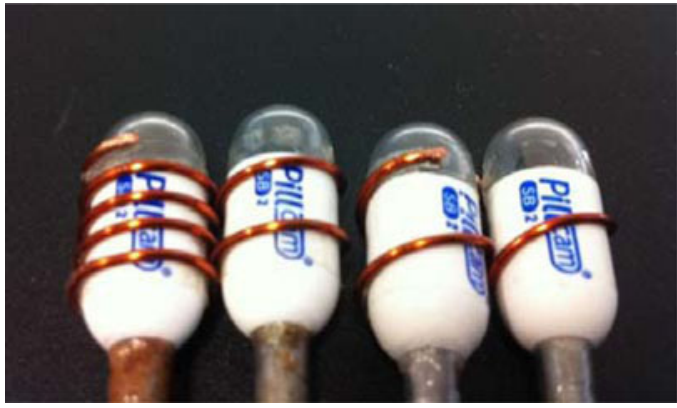

Fig. 1. Capsules with different spiral structures.

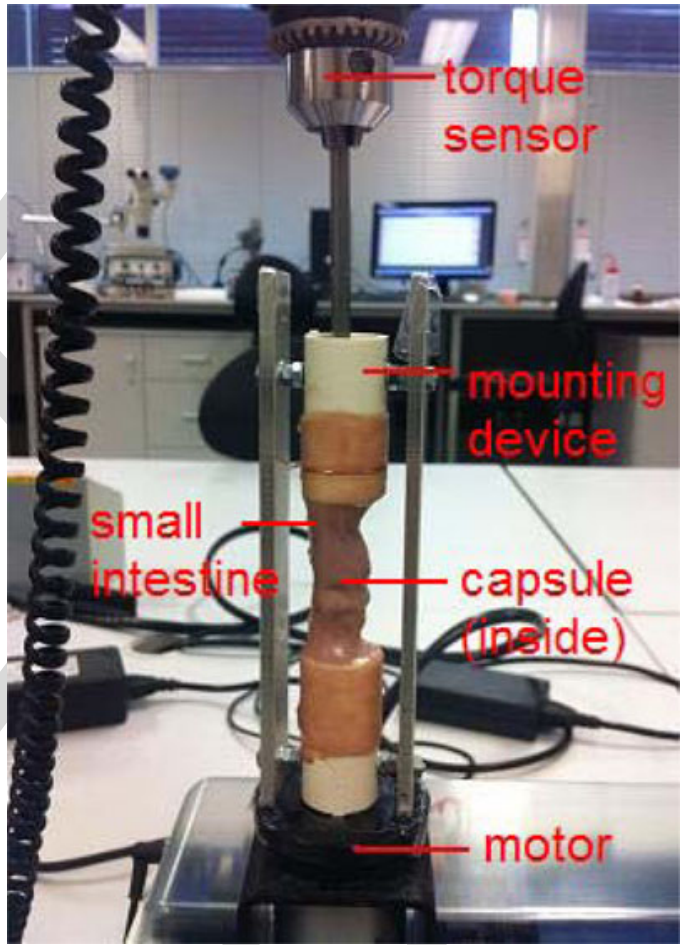

Fig. 2. General view of the experimental setup.

mounted on a custom-built device, comprised of two coaxial plastic tubes and two aluminum bars for supporting. Then, this device was attached to an electric motor, whose rotating speed was adjusted by a Labview program sending commands via a data-acquisition (DAQ) board and a control module. In order to avoid the influence from the gravity, the devices were lined up vertically. The general view of the experimental setup is illustrated in Fig. 2.

\section{B. Experimental Procedures}

The intestinal specimens, kept in a refrigerator, were unfrozen a few hours before the experiments. Then, they were immersed in a jar of physiological saline, which was helpful to prevent tissue rupture. One intestinal segment and one capsule were placed as shown in Fig. 2 each time. The small intestine was rotated at a constant speed for several seconds by the electric motor so that the real-time frictional torque could be measured by the torque sensor. An initial test was carried out with one specimen 


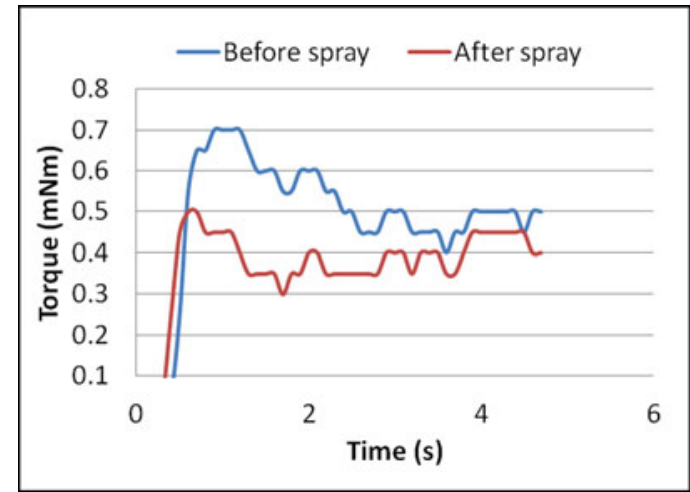

Fig. 3. Initial test with the small intestine Sample 1.

(Sample 1). Afterward, a set of tests were performed with the other two intestinal specimens successively. The internal diameters were $10.9 \mathrm{~mm}$ (Sample 2) and $11.1 \mathrm{~mm}$ (Sample 3), respectively. Both of them had the thickness of $1 \mathrm{~mm}$. For each set of test, the rotating frequencies in the range of $0.5-3 \mathrm{~Hz}$ were applied.

The small intestine would be dried out fast once it was displaced out of the fluid. However, the saline could not be sprayed on the segment during the experiment since it would change the frictional properties of the intestine, as explained in the following section. Therefore, in order to keep the experimental conditions as consistent as possible, the tests were finished in a short time for either Sample 2 or Sample 3. Additionally, after each measurement, the sample was inspected whether a slight twist occurred due to the contact with the capsule. If so, a quick and simple treatment would be conducted manually to bring it back to the original state.

When all the tests for one specimen were finished, the intestinal tube was cut open and flattened on a table immediately. By using a force sensor and a smooth capsule with the mass of $3.98 \mathrm{~g}$, the COF was determined for each intestinal specimen by the following equation:

$$
\mu=\frac{f}{m g}
$$

where $f$ is the force to overcome the friction, and $m$ is the mass of the pulled capsule, and $g$ is the gravity of earth.

All the experiments were carried out in an air-conditioned space, which maintained the room temperature at $25^{\circ} \mathrm{C}$.

\section{Results and Discussions}

Fig. 3 shows the torque measurements with the capsule No. 2 (helical angle $=10^{\circ}$ ) and the small intestine Sample 1 under the rotating frequency of $1 \mathrm{~Hz}$. Fig. 4 shows the results with two different segments. From Fig. 4, a slightly higher static friction occurs first and then a relatively steady dynamic friction can be observed. After spraying some saline on Sample 1, a reduction in the frictional resistance is quite apparent, implying the change of the tissue's biomechanical and tribological properties due to the absorption of liquid. Therefore, humidifying the intestine during

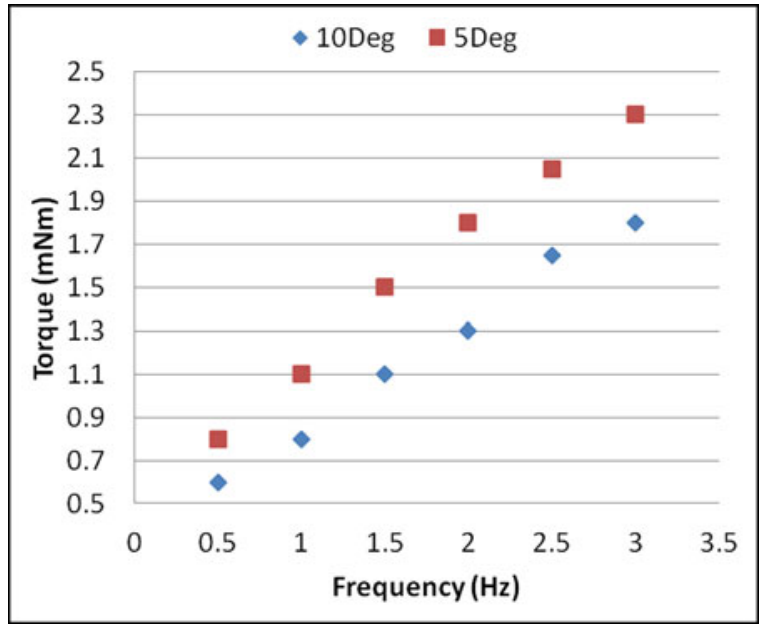

Fig. 4. Torque Measurement with the small intestine Sample 2.

the tests is inappropriate for the consistence of the experimental environment.

In the tests with Sample 2 and Sample 3, the measurements of dynamic friction are compared to each other as different combinations of capsule and intestinal specimens as well as frequency were adopted.

Sample 2 was tested with the capsules No. 1 (helical angle = $5^{\circ}$ ) and No. 2, respectively. The results are shown in Fig. 4. From the graph, it is seen that the frictional torque increases as the rotation frequency rises, indicating the rotational resistance has a relationship with the rate of strain of the small intestine. This dependence reveals the viscoelasticity of the GI tract to some extent. Due to the introduction of the spiral, the cross section of the capsule in the lateral direction is raised, increasing the deformation of the intestine. This increase becomes larger when the helical angle gets smaller. Therefore, when the number of spiral is the same, a capsule with a smaller helical angle causes more strain in the intestine, and consequently, confronts a higher frictional resistance. In this case, from 0.5 to $3 \mathrm{~Hz}$, the capsule No. 2 (helical angle $=10^{\circ}$ ) causes a torque in the range of $0.6-1.8 \mathrm{mN} \cdot \mathrm{m}$ while the capsule No. 1 (helical angle $=5^{\circ}$ ) results in the magnitude between 0.8 and $2.3 \mathrm{mN} \cdot \mathrm{m}$. At low frequencies, the torque is almost proportional to the frequency and the proportionality constant is bigger when the helical angle is smaller.

Sample 3 was tested with all the four capsules one by one. The torque measurements are presented in Fig. 5. For the same combinations of capsule, specimen, and frequency, the results are slightly different from those of Sample 2 due to the discrepancy of two intestinal specimens' conditions. However, the magnitude of the resistant feature is still in the same order. Moreover, the trend is nearly identical to that of Sample 2.

The COFs of two intestinal segments were also determined by (1) for the parameter identification of the analytical model proposed in the following section. Fig. 6 shows a sample measurement of the force versus time for the smooth capsule to overcome the friction on the cut-open and flattened small intestine (Sample 2). Since the mass of the capsule is already known, 


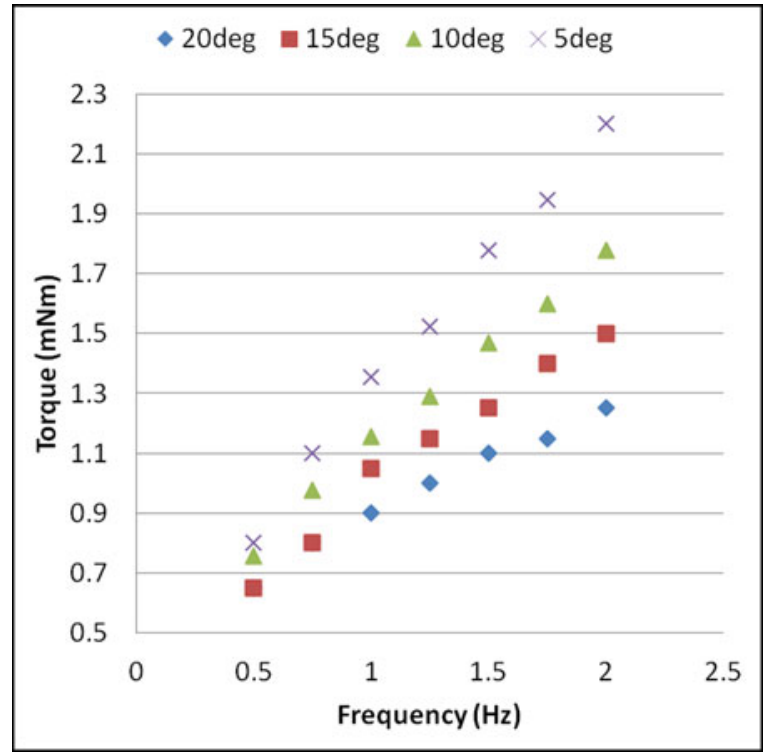

Fig. 5. Torque Measurement with the small intestine Sample 3.

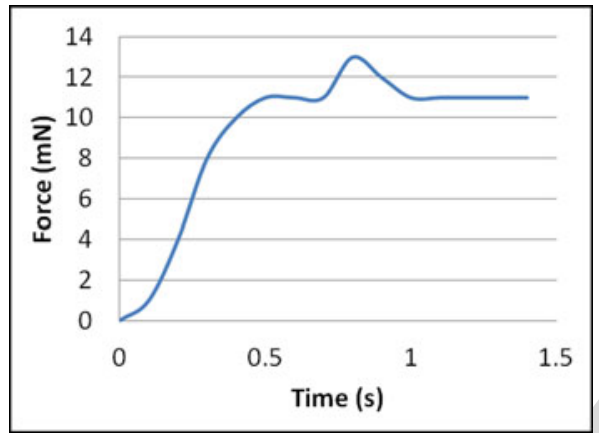

Fig. 6. Force history for a smooth capsule to overcome the friction on the cut-open and flattened small intestine Sample 2.

the COF for Sample 2 can be calculated as 0.28 . The same method is used to find the COF for Sample 3, which is equal to 0.51 . We postulate that the difference between these COFs can be due to different wetness conditions on the inner surfaces of these two samples. The inner surface of Sample 2 was slightly humidified with the saline before it was tested while the same treatment was not applied to Sample 3. As reported before, a small amount of lubrication could change the friction in a small intestine dramatically [17].

\section{MODELING}

\section{A. Viscoelasticity Model}

Generally, the relationship between stress and strain of a viscoelastic material can be illustrated by a generalized Maxwell model including multiple viscous dashpots and elastic springs [22]. Fig. 7 shows a five-element linear viscoelastic model, which is employed to describe the mechanical behavior of the small intestine in this study. The constitutive equation is ex-

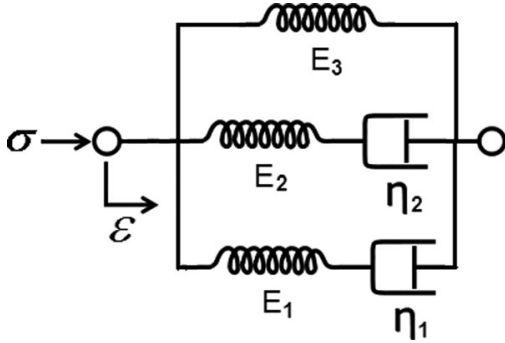

Fig. 7. Five-element viscoelasticity model.

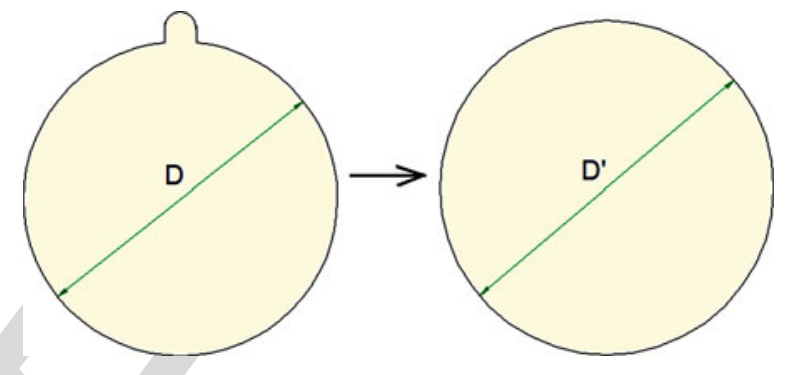

Fig. 8. Conversion of the inner intestinal wall's profile.

pressed as follows:

$$
\sigma(t)=\varepsilon(t)\left[E_{1} \exp \left(-\frac{t E_{1}}{\eta_{1}}\right)+E_{2} \exp \left(-\frac{t E_{2}}{\eta_{2}}\right)+E_{3}\right]
$$

where $\sigma$ and $\varepsilon$ denote the stress and the strain at the time $t, 248$ respectively, and $E_{1}, E_{2}$, and $E_{3}$ are the elastic modulus of 249 springs, and $\eta_{1}$ and $\eta_{2}$ represent the viscosities of dashpots.

\section{B. Analytical Model for Rotational Resistance}

As discussed earlier, attaching a spiral structure on the surface 252 increases the cross section of the capsule in its lateral direction. 253 In Fig. 8, the left-hand side profile describes the inner wall's 254 cross-sectional profile of the small intestine after its deforma- 255 tion due to the insertion of the spiral-type capsule. The bulge 256 results from the spiral structure and the parameter $D$ indicates 257 the diameter of the cylindrical part of Pillcam SB2 capsule 258 $(11 \mathrm{~mm})$. To simplify the analysis, as the capsule rotates, the 259 contour is converted to a circular geometry whose perimeter is 260 comparable to the total length of the original one, which means 261 the circumferential extension of the intestinal tract is kept the 262 same. The right-hand side profile in Fig. 8 shows the converted 263 circular profile of the deformed intestine's inner surface with 264 a new diameter of $D^{\prime}$. Since the spiral is only wounded on the 265 cylindrical part, $D^{\prime}$ is just applied to calculate the strain of tissue 266 for this area. The practical dimensions are used for the frontal 267 and rear parts.

In addition to this geometrical simplification, a few assump- 269 tions are made to develop the model. 270

1) The intestinal tissue is an isotropic and incompressible 271 material.

2) The volume and the wall thickness of the small intestine 273 are constant. 


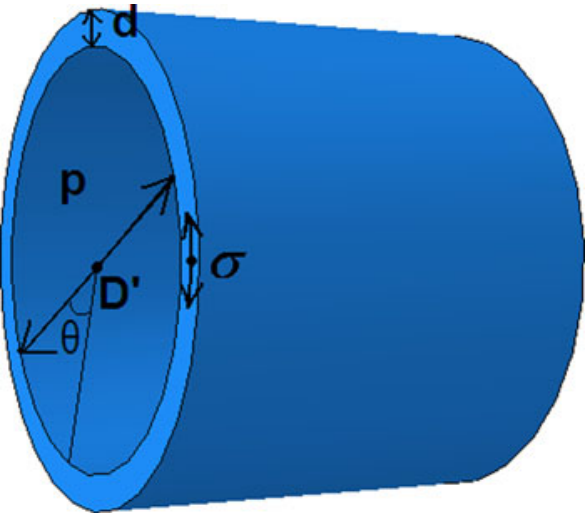

Fig. 9. Pressure vessel for the intestinal tract modeling.

3) The deformation of the intestine corresponds to the contact area and is symmetrical toward the radial direction after the geometrical simplification.

In order to analyze the normal load exerted on the capsule, the internal pressure generated by the intestinal tract due to circumferential extension has to be determined. Therefore, the intestine is modeled as a cylindrical pressure vessel [23] shown in Fig. 9. The pressure can be calculated from

$$
p(\theta)=\frac{\sigma(\theta) 2 d}{D^{\prime}}
$$

where $d$ is the thickness of the small intestine, and $D^{\prime}$ is the diameter of the converted inner intestinal surface's profile, and $\sigma$ and $p$ are the circumferential stress and the corresponding pressure at the azimuth $\theta$, respectively.

For a rotational movement, the time $t$ is the division of the azimuth $\theta$ by the angular velocity $\omega$. Hence, (2) can be written to express the relationship between the stress $\sigma$ and the rotating frequency $f$ as follows:

$$
\begin{aligned}
\sigma(\theta) & =\varepsilon(\theta)\left[E_{1} \exp \left(-\frac{\theta E_{1}}{\omega \eta_{1}}\right)+E_{2} \exp \left(-\frac{\theta E_{2}}{\omega \eta_{2}}\right)+E_{3}\right] \\
\omega & =2 \pi f .
\end{aligned}
$$

For every lateral cross section, the circumerferenital strain due to capsule insertion is determined by

$$
\varepsilon=\frac{D_{\text {after }}-D_{\text {before }}}{D_{\text {before }}+d}
$$

where $D_{\text {before }}$ and $D_{\text {after }}$ are the inner diamters of the intestinal tract before and after deformation, respectively. For the middle part with the spiral, $D_{\text {after }}$ is equal to $D^{\prime}$. For the frontal and rear parts without the spiral, only the contact areas are taken into account. Since these parts are semispheres, $D_{\text {after }}$ varies with the axial position and can be calculated with the spherical radius, which is $5.5 \mathrm{~mm}$.

The total normal load for one cross section can be obtained by using (3) to integrate the pressure along the circumference. Once the frictional coefficient $\mu$ is evaluated, the circumferential friction can be calculated with Coulomb's law of friction. Since the distance between the force and rotating axis is fixed, the
TABLE I

IDENTIFIED PARAMETERS FOR THE SMALL INTESTINE SAMPLE 2

\begin{tabular}{cc}
\hline \hline Parameters & Numerical Values \\
\hline $\mathrm{E}_{1}(\mathrm{kPa})$ & 0.12 \\
$\mathrm{E}_{2}(\mathrm{kPa})$ & 18.64 \\
$\mathrm{E}_{3}(\mathrm{kPa})$ & 0.0122 \\
$\eta_{1}(\mathrm{kPa} \mathrm{s})$ & 43.51 \\
$\eta_{2}(\mathrm{kPa} \mathrm{s})$ & 0.2213 \\
\hline \hline
\end{tabular}

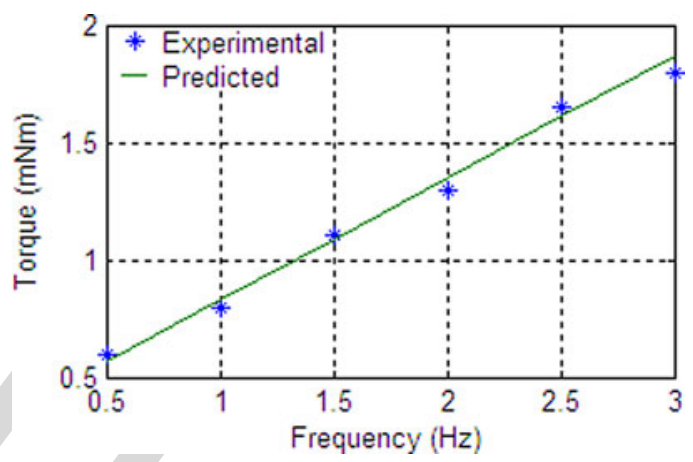

Fig. 10. Variation of the frictional torque with the capsule No. 2 rotating inside the small intestine Sample 2.

rotational resistance can be solved in the form of a resistive 305 torque. The equations are expressed as follows:

$$
\begin{aligned}
& f=\mu \int p(\theta) d \theta \\
& \tau=\frac{f D_{\text {after }}}{2} .
\end{aligned}
$$

where $f$ is the frictional force in the circumferential direction 307 and $\tau$ is the resistant torque as a result of rotation.

\section{Parameter Identification and Model Validation}

To identify the elastic modulus and viscosities in the model, a 310 nonlinear least square optimization process is employed in this 311 study. Based on the nonlinear relationship between the frictional 312 torque and the rotating frequency, these material parameters 313 were estimated by means of minimizing the summed square of 314 the error vector with the experimental data presented earlier. A 315 numerical search method, which is the interior-reflective New- 316 ton algorithm, was used to solve the problem. It acquires the 317 approximate solution of a system by utilizing the method of 318 preconditioned conjugate gradients at each iteration. It is sug- 319 gested that this method is efficient for nonlinear optimization 320 problems [24].

For the small intestine Sample 2, the measurements with the capsule No. 2 (helical angle $=10^{\circ}$ ) was used to estimate the parameters. The numerical values are listed in Table I. The experimental and predicted frictional torques corresponding to the frequencies are shown in Fig. 10.

The measurements with the capsule No. 1(helical angle $=5^{\circ}$ ) was used to validate the model for the small intestine Sample 2 shown in Fig. 11. With reference to these results, the estimated values are quite consistent to the experimental results, indicating that the analytical model is effective enough to predict the 


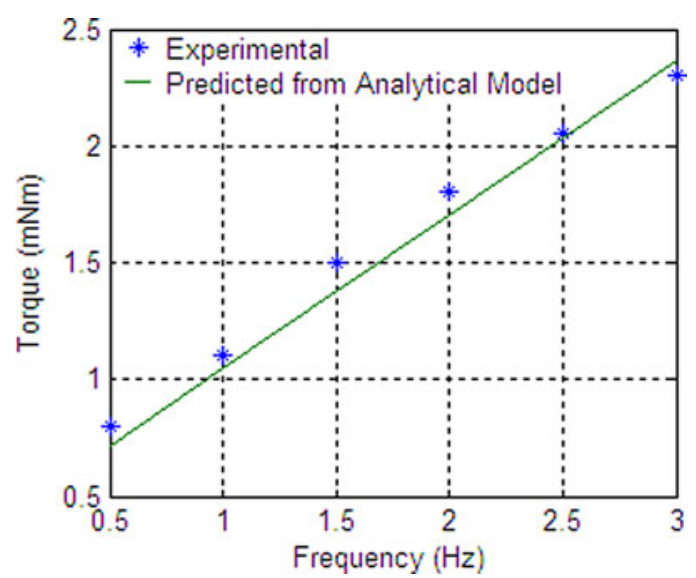

Fig. 11. Validation for the small intestine Sample 2.

TABLE II

IDENTIFIED PARAMETERS FOR THE SMALL INTESTINE SAMPLE 3

\begin{tabular}{cc}
\hline \hline Parameters & Numerical Values \\
\hline $\mathrm{E}_{1}(\mathrm{kPa})$ & 0.0348 \\
$\mathrm{E}_{2}(\mathrm{kPa})$ & 0.7722 \\
$\mathrm{E}_{3}(\mathrm{kPa})$ & 0.0448 \\
$\eta_{1}(\mathrm{kPa} \mathrm{s})$ & 9.491 \\
$\eta_{2}(\mathrm{kPa} \mathrm{s})$ & 0.2489 \\
\hline \hline
\end{tabular}

rotational resistance resulting from the spiral-type capsule rotating inside the small intestine Sample 2.

Though the trend of mechanical behavior is identical, the specific viscoelasticity of the intestinal tract may be different from one sample to another due to many experimental factors such as the freezing period, humidification level, and even variation in the tissue's microstructure. Therefore, the nonlinear least square optimization was repeated to identify the elastic modulus and viscosities of the model for the small intestine Sample 3. The measurements with the capsule No. 3 (helical angle $=15^{\circ}$ ) was employed. The estimated parameters are listed in Table II. The experimental and predicted resistant torque values are presented in Fig. 12. These results also demonstrate the efficacy of the analytical model in predicting the mechanical torque needed to overcome resistive effects associated with the viscoelastic intestine environment.

To evaluate the accuracy of the model for Sample 3, the measurements with other three capsules were compared to the predicted values with the estimated parameters, as illustrated in Fig. 13. With reference to these results, the model performs well when predicting the rotational resistance (i.e., torque) of the capsule No. 2 (helical angle $=10^{\circ}$ ). Though the prediction's accuracy is a bit lower for the other two capsules, the prediction is still in a reasonable range. We postulate that the discrepancy is possibly due to the effect of the stress concentration around the spiral structure, which is neglected in this model. In addition, the condition of the small intestine Sample 3 might slightly change during the tests due to a relatively longer experimental time compared to that with Sample 2.

From the estimated parameters for Sample 2 and Sample 3, it can be seen that the mechanical properties of different segments

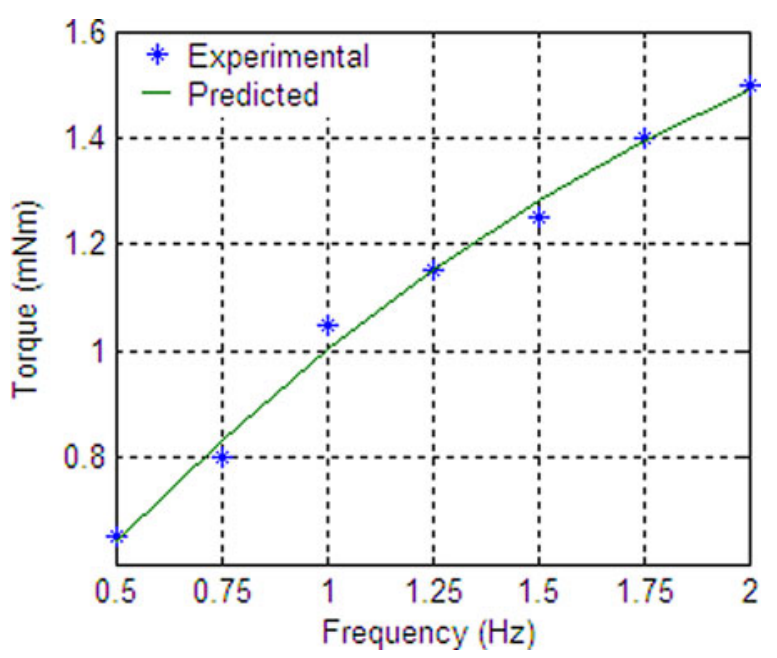

Fig. 12. Variation of the frictional torque with the capsule No. 3 rotating inside the small intestine Sample 3.

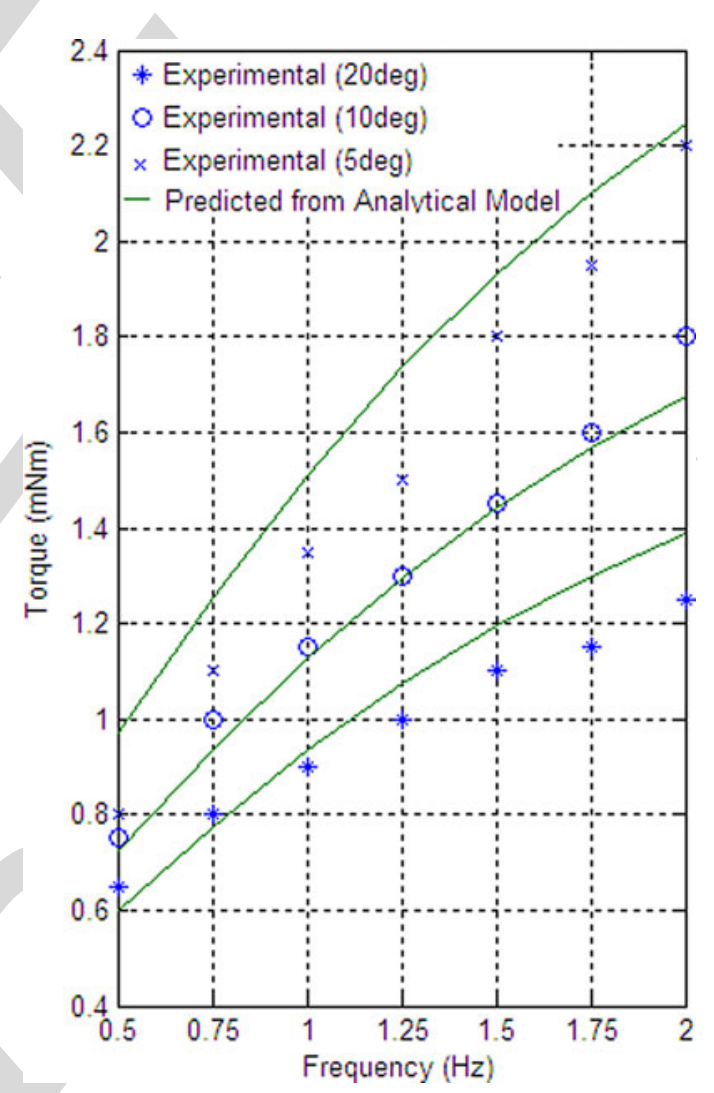

Fig. 13. Validation for the small intestine Sample 3.

(i.e., samples) exhibit some variance as expected, though they 363 both show the viscoelastic properties and exert the rotational 364 resistance in the same order of magnitude on the inserted capsule 365 due to the deformation and rotation.

\section{CONCLUSION}

The rotational resistance of a spiral-type capsule rotating in- 368 side the small intestine is investigated by both in vitro exper- 369 iments and mathematical modeling and analysis, on which a 370 
limited literature is available. The experimental results show the viscoelastic nature of the intestinal tissue and the effects of various spiral structures and rotating speeds. At low rotating frequencies $(0.5-3 \mathrm{~Hz})$, a capsule $(\phi 11 \times 26 \mathrm{~mm})$ wounded with a 1-mm-high spiral works against a frictional torque varying from $0.5 \mathrm{mN} \cdot \mathrm{m}$ to several $\mathrm{mN} \cdot \mathrm{m}$. The torque increases with the increase of the frequency. Due to the same number of spiral in the tests, the helical structure with a smaller helical angle raises more in the lateral cross section of the robotic capsule, which results in more circumferential deformation of the small intestine and more rotational resistance. Viscoelastic properties of the "real" intestine are identified using a nonlinear optimization method. The validation results show that the proposed torque model is reasonably effective to estimate the rotational resistive torque of the small intestine. For different intestinal samples, though the rotational resistance is in the same order of magnitude, their biomechanical and tribological properties may show some variance due to the different conditions such as the duration of freezing time, intestine size, capsule weight, and humidification level. Both the experimental and modeling work provide a useful reference to characterize the required torque for a spiral-type capsule and, therefore, helps to undertake the design and optimization of the microrobots for medical use in the GI tract. However, before such a spiral-type robot is used in a real GI tract, in vivo experiments should be conducted in a highly unstructured, slippery, deformable, and nonsmooth environment to obtain a more accurate estimate of the rotational resistance.

Further work will aim to conduct more experiments with various intestinal specimens and improve the analytical model so that the stress concentration and viscoelastic properties due to variable-size spiral structures can be taken into account. Furthermore, some addition will be made to the current experimental setup so that a rotational and translational movement can be allowed between the capsule and the inner wall of the small intestine. The rotational resistance with different translational speed of the capsule will be investigated and compared to the current results.

\section{ACKNOWLEDGMENT}

The authors would like to thank Given Imaging Pty. Ltd, Sydney, Australia, for providing the endoscopic capsules used in this study.

[1] R. Eliakim, "Video capsule endoscopy of the small bowel," Curr. Opin. Gastroenterol., vol. 26, pp. 129-133, 2010.

[2] M. Waterman and R. Eliakim, "Capsule enteroscopy of the small intestine," Abdominal Imag., vol. 34, pp. 452-458, 2009.

[3] H. Niemenmaa, T. Mäkelä, A. Jussilia, I. Krekelä, M. Voutilainen, H. Björknäs, A. Hirvioja, K. Kaukinen, and P. Collin, "The diagnostic value of video capsule endoscopy," Eur. J. Internal Med., vol. 21, pp. 383-385, 2010.

[4] A. Moglia, A. Menciassi, P. Dario, and A. Cuschieri, "Capsule endoscopy: Progress update and challenges ahead," Nat. Rev. Gastroenterol. Hepatol., vol. 6, pp. 353-362, 2009.

[5] A. Moglia, A. Menciassi, and P. Dario, "Recent patents on wireless capsule endoscope," Recent Patents Biomed. Eng., vol. 1, pp. 24-33, 2008.

[6] J. L. Toennies, G. Tortora, M. Simi, P. Valdastri, and R. J. Webster III, "Swallowable medical devices for diagnosis and surgery: The state of the art," Proc. IMech C, J. Mech. Eng. Sci., vol. 224, pp. 1397-1414, 2010.

[7] B. Kim, S. Lee, J. H. Park, and J. O. Park, "Design and fabrication of a locomotive mechanism for capsule-type endoscopes using shape memory alloys (SMAs)," IEEE/ASME Trans. Mechatronics, vol. 10, no. 1, pp. 7786, Feb. 2005.

[8] M. Simi, P. Valdastri, C. Quaglia, A. Menciassi, and P. Dario, "Design, fabrication, and testing a capsule with hybrid locomotion for gastrointestinal tract exploration,” IEEE/ASME Trans. Mechatronics, vol. 15, no. 2 , pp. 170-180, Apr. 2010.

[9] F. Carpi, N. Kastelein, M. Talcott, and C. Pappone, "Magnetically controllable gastrointestinal steering of video capsules," IEEE Trans. Biomed. Eng., vol. 58, no. 2, pp. 231-234, Feb. 2011.

[10] F. Carpi, "Magnetic capsule endoscopy: The future is around the corner," Expert Rev. Med. Devices, vol. 7, pp. 161-164, 2010

[11] M. Gao, C. Hu, Z. Chen, H. Zhang, and S. Liu, "Design and fabrication of a magnetic propulsion system for self-propelled capsule endoscope," IEEE Trans. Biomed. Eng., vol. 57, no. 12, pp. 2891-2902, Dec. 2010.

[12] M. Sendoh, K. A. Yamazaki, A. Chiba, M. Soma, K. Ishiyama, an K. I. Arai, "Spiral type magnetic micro actuators for medical applications," in Proc. Int. Symp. Micro-NanoMechatron. Human Sci., 2004 pp. 319-324.

[13] Y. Zhang, S. Jiang, X. Zhang, X. Ruan, and D. Guo, "A variable-diameter capsule robot based on multiple wedge effects," IEEE/ASME Trans. Mechatronics, vol. 16, no. 2, pp. 241-254, Apr. 2011.

[14] N. K. Baek, I. H. Sung, and D. E. Kim, "Frictional resistance characteristics of a capsule inside the intestine for microendoscope design," Proc Instn Mech. Eng. J, Eng. Med., vol. 218, pp. 193-201, 2004.

[15] J. S. Kim, I. H. Sung, Y. T. Kim, E. Y. Kwon, D. E. Kim, and Y. H. Jang, "Experimental investigation of frictional and viscoelastic properties of intestine for microendoscope application," Tribol. Lett., vol. 22, pp. $143-$ 149,2006

[16] J. S. Kim, I. H. Sung, Y. T. Kim, and Y. H. Jang, "Analytical mode development for the prediction of the frictional resistance of a capsule endoscope inside an intestine," Proc. Instn Mech. Engrs PartH: J. Eng. Med., vol. 221, pp. 837-845, 2007.

[17] X. Wang and M. Q. H. Meng, "An experimental study of resistant properties of the small intestine for an active capsule endoscope," Proc. Instn Mech. Engrs PartH: J. Eng. Med., vol. 224, pp. 107-118, 2010.

[18] K. D. Wang and G. Z. Yan, "Research on measurement and modeling of the gastro intestine's frictional characteristics," Meas. Sci. Technol., vol. 20, p. 015803 (6pp), 2009.

[19] B. S. Terry, A. B. Lyle, J. A. Schoen, and M. E. Rentschler, "Preliminary mechanical characterization of the small bowel for in vivo robotic mobility," ASME J. Biomech. Eng., vol. 133, pp. 091010-1-091010-7, 2011.

[20] C. Bellini, P. Glass, M. Sitti, and E. S. Di Martino, "Biaxial mechanical modeling of the small intestine," J. Mech. Behav. Biomed. Mater., vol. 4 pp. 1727-1740, 2011.

[21] H. D. Hoeg, A. B. Slatkin, and J. W. Burdick, "Biomedical modeling of the small intestine as required for the design and operation of a robotic endoscope," in Proc. IEEE Int. Conf. Rob. Autom., 2000, pp. 1599-1606.

[22] W. Flugge, Viscoelasticity. Berlin, Germany: Springer-Verlag, 1975.

[23] J. M. Gere, Mechanics of Materials, 7th ed. Toronto, ON, Canada: Cengage Learning, 2009.

[24] G. Alici, "An effective modeling approach to estimate nonlinear bending behavior of cantilever type conducting polymer actuators," Sens. Actuators B, Chem., vol. 141, pp. 284-292, 2009.

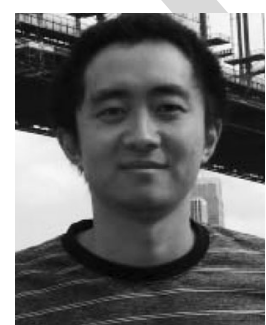

Hao Zhou received the B.S. degree in building environment and facility engineering from Tongji University, Shanghai, China, in 2004, the M.S. degree in mechanical engineering from the University of Queensland, Australia, in 2008, and the M.S. degree in engineering practice (mechanical) from the University of Wollongong, Wollongong, Australia, in 2009, where he is currently working toward the Ph.D. degree.

His research interests include simulations an analysis of electromagnetics, simulations and analysis of biomechanics of the small intestine, mechanics of viscoelastic materials, and active locomotion of wireless capsule endoscopy. 


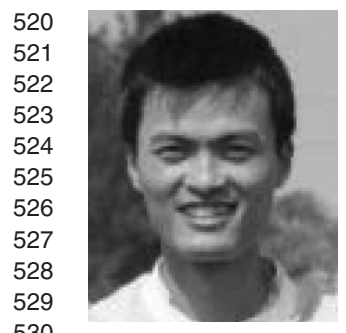

Gursel Alici received the Ph.D. degree in robotics from the Department of Engineering Science, Oxford University, Oxford, U.K., in 1994.

$\mathrm{He}$ is currently a Professor at the University of Wollongong, Wollongong, Australia, where he is the Head of the School of Mechanical, Materials and Mechatronic Engineering. His current research interests include intelligent mechatronic systems involving mechanisms/serial/parallel robot manipulators, micro/nano robotic systems for medical applications, and modeling, analysis, characterization, and control of conducting polymers as macro/micro/nanosized actuators and sensors for robotic and bioinspired applications. He has produced more than 200 refereed publications in his areas of research.

Dr. Alici was a Technical Editor of the IEEE/ASME TRANSACTIONS ON MECHATRONICS during 2008-2012, and is a member of the Mechatronics National Panel formed by the Institution of Engineers, Australia. He was the recipient of the Outstanding Contributions to Teaching and Learning Award from the University of Wollongong, in 2010.

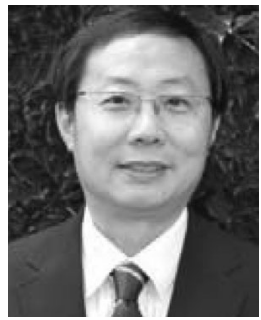

Weihua Li received the B.Eng. degree in 1992 and 532 the M.Eng. degree in 1995 both from the University 533 of Science and Technology of China and the Ph.D. de- 534 gree from Nanyang Technological University (NTU), 535 Singapore, in 2001 .

After two year's postdoctoral training at NTU, he 537 joined the University of Wollongong, Wollongong, 538 Australia, as a Full-Time Academic, where he is cur- 539 rently working as a Discipline Advisor for Mecha- 540 tronic Engineering. He has multidisciplinary areas of 541 expertise, including smart materials and structures, 542 microfluidics, intelligent mechatronics, and dynamics and vibration control. He 543 has coauthored more than 160 articles, and delivered many plenary or invited 544 talks at various international conferences.

Dr. Li is currently serving as an Editorial Board member for more than eight 546 international journals.

Trung Duc Than received the B.S. degree in electrical engineering from the Hanoi University of Technology, Hanoi, Vietnam, in 2007, and the M.S. degree in electrical and computer engineering from the University of Wollongong, Wollongong, N.S.W., Australia, in 2010, where he is currently working toward the Ph.D. degree in the School of Mechanical, Materials, and Mechatronic Engineering.

His research interest includes the development of localization and control systems for robotic applications.

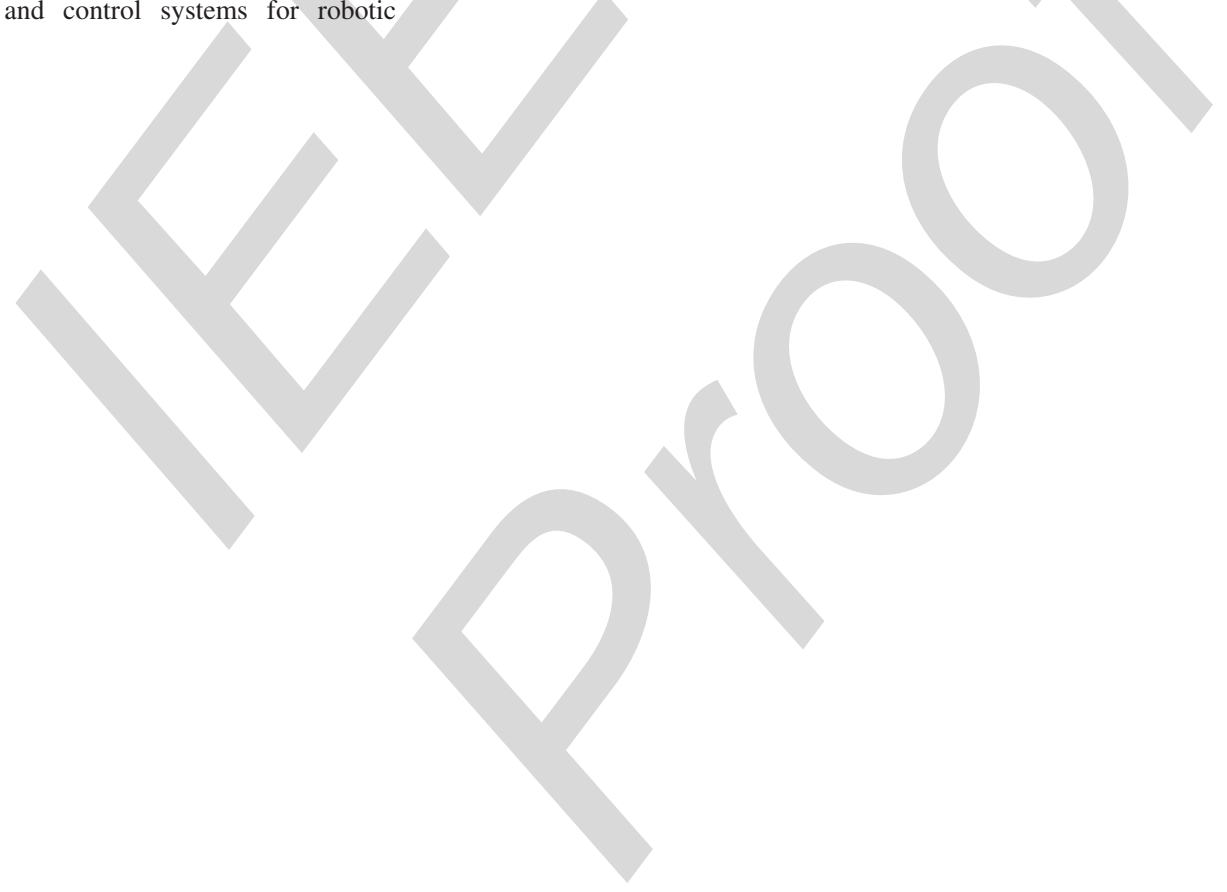


Q1: Author: Please supply index terms/keywords for your paper. To download the IEEE Taxonomy go to 550 http://ieee.org/documents/2009Taxonomy_v101.pdf. 551

Q2: Author: Please verify the affiliation as set.

Q3. Author: Please provide the full page range in reference [18]. 